TRANSACTIONS OF THE

AMERICAN MATHEMATICAL SOCIETY

Volume 355, Number 12, Pages 4847-4863

$\mathrm{S} 0002-9947(03) 03112-\mathrm{X}$

Article electronically published on July 28, 2003

\title{
TIGHT SURFACES IN THREE-DIMENSIONAL COMPACT EUCLIDEAN SPACE FORMS
}

\author{
MARC-OLIVER OTTO
}

\begin{abstract}
In this paper we define and discuss tight surfaces - smooth or polyhedral - in three-dimensional compact Euclidean space forms and prove existence and non-existence results. It will be shown that all orientable and most of the non-orientable surfaces can be tightly immersed in any of these space forms.
\end{abstract}

\section{INTRODUCTION}

In the early 1960s Nicolaas Kuiper defined and studied tight immersions of surfaces in Euclidean three-space $\mathbb{E}^{3}$ in 13 and 14. Let $M$ be a smooth $\left(C^{\infty}\right)$-surface with Euler characteristic $\chi(M)$ immersed in Euclidean three-space. By definition, the total absolute curvature of $M$ is

$$
\tau(M)=\int_{M} \frac{|K| d o}{2 \pi}
$$

where $K$ denotes the Gaussian curvature and $d o$ is the absolute value of the exterior 2-form which represents the volume. With Gauss-Bonnet's theorem and the knowledge of inequality (1.1) for the integral over all points of positive Gaussian curvature $K$,

$$
\int_{K>0} \frac{K d o}{2 \pi} \geq 2
$$

it follows (see [13]) that inequality (1.2) holds for immersions of closed surfaces in Euclidean three-space

$$
\tau(M)=\int_{M} \frac{|K| d o}{2 \pi} \geq 4-\chi(M) .
$$

If equality occurs in (1.2), the immersion $f: M \rightarrow \mathbb{E}^{3}$ is called tight, which means that the surface has minimal total absolute curvature. Tightness is a generalization of the notion of convexity. In some sense, it means that a surface is embedded or immersed "as convexly as possible" with respect to its topological properties. With the introduction of the two-piece-property (TPP), which is equivalent to equality in (1.2) for surfaces in $\mathbb{E}^{3}$, it was possible to examine tightness not only for smooth surfaces but also for polyhedral ones as was done by Banchoff in [1]. For all surfaces — smooth or polyhedral - it is known whether they can be immersed or embedded

Received by the editors May 28, 2001 and, in revised form, May 29, 2002.

2000 Mathematics Subject Classification. Primary 53C42; Secondary 57M50.

Key words and phrases. Tight immersions, tight embeddings, Euclidean space forms, total absolute curvature. 
tightly in three-space or not. A good collection of the known results and a huge reference list on tightness can be found in [3].

In the present paper we transfer these results to the case where the surfaces - smooth or polyhedral - are immersed in three-dimensional compact Euclidean space forms $(C E S)$. By definition, a $C E S$ is the compact quotient $\mathbb{R}^{3} / \Gamma$ where $\Gamma$ is a freely acting discrete subgroup of the Euclidean group. Now, the problem of classifying the $C E S$ is reduced to searching for these groups. Bieberbach's theorems show that there are only finitely many in each dimension, and therefore, also a finite number of $C E S$. With the help of this, Hantzsche and Wendt classified the CES in [10] and proved that there are only six orientable and four non-orientable ones in dimension three.

Having this result we are able to examine properties of surfaces immersed in these $C E S$. We get similar inequalities for their total absolute curvature as in the case of Euclidean three-space. The immersions for which equality occurs in these will be called tight. Then existence and non-existence results of tightly immersed surfaces in $C E S$ will be proved.

We show in Theorem 3.1 and Theorem 3.4 that all orientable surfaces and all non-orientable surfaces with even Euler characteristic less than zero admit a tight immersion in any $C E S$. Theorem 3.5 proves the existence of a tight immersion of all surfaces with odd Euler characteristic less than -3 in any $C E S$ while Theorem 3.2 deals with the Klein bottle: existence of a tight immersion in all $C E S$ but the three-torus and one other orientable $C E S$ is proved. The projective plane with two handles admits at least a tight polyhedral immersion in any $C E S$ as shown in Theorem 3.6. Theorem 3.8 and Theorem 3.9 prove the non-existence of tight immersions of the projective plane in any $C E S$ and of the Klein bottle in the three-torus and one other orientable $C E S$. The only open cases are therefore the projective plane with one handle and a smooth tight immersion of the projective plane with two handles in any $C E S$.

\section{Definitions}

As stated in the introduction, the definition and the classification of $C E S$ can be found in [10] or in a modern way in [16] where we adopted our notation. We denote the different $C E S$ by $\mathbb{R}^{3} / \Gamma_{i}, i=1, \ldots, 6$, for orientable $C E S$, by $\mathbb{R}^{3} / \mathfrak{B}_{\text {j }}$, $j=1, \ldots, 4$, for non-orientable $C E S$ and by $\mathbb{R}^{3} / \Gamma$ if we mean both of them. The exact definitions of the various groups $\Gamma_{i}$ and $\mathfrak{B}_{\mathfrak{j}}$ can also be found in [16] (section 3.5), however, we omit them here.

In the classical reference one can find diagrams of the group actions. We extend these to diagrams of the fundamental domains of the ten different groups $\Gamma$. The resulting diagrams of the fundamental domains of the groups $\Gamma_{i}$ which represent the orientable $C E S$ can be seen in Figure 1. The arrows mark the way the edges are identified. They imply the identifications of the faces, where we are only allowed to identify those faces whose edges are oriented in the same way. These identifications emerge from the study of the group actions.

A surface $M$ is a compact and connected two-dimensional manifold without boundary. $M$ is embedded into a $C E S$ if it is homeomorphic to a subset of a $C E S$ and immersed into a $C E S$ by a continuous mapping $f: M \rightarrow \mathbb{R}^{3} / \Gamma$ if $f$ is locally injective. If $M$ is of differentiability class $C^{\infty}$, we will speak of a smooth surface. This together with a $C^{\infty}$-mapping shall be a smooth immersion of $M$. A polyhedral 


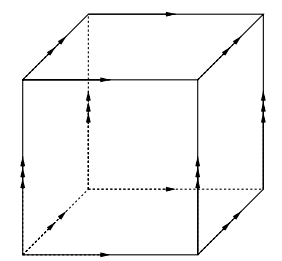

(a) Fundamental domain of $\Gamma_{1}$

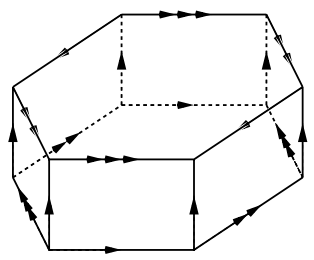

(c) Fundamental domain of $\Gamma_{3}$

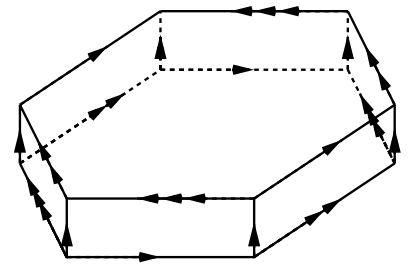

(e) Fundamental domain of $\Gamma_{5}$

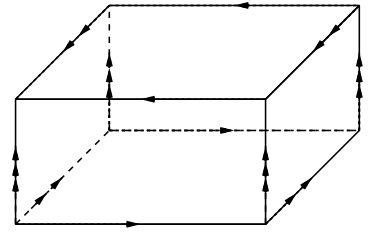

(b) Fundamental domain of $\Gamma_{2}$

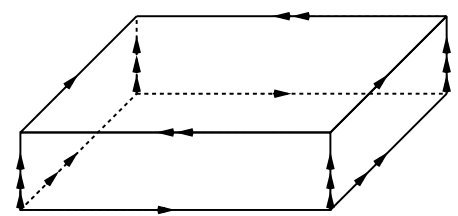

(d) Fundamental domain of $\Gamma_{4}$

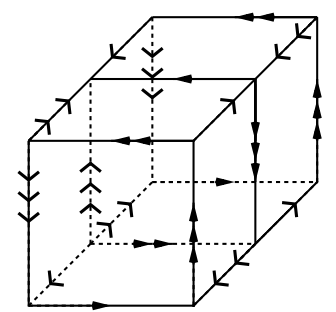

(f) Fundamental domain of $\Gamma_{6}$

Figure 1. Models of the orientable $C E S$

surface $M \subset \mathbb{R}^{3} / \Gamma$ is a surface embedded in a $C E S$ such that $M$ is a finite union of planar polygons where any two of them have no interior points in common and nonadjacent faces have no points in common. By subdivision, one can always assume that the polygons are convex. Then the intersection of two such convex polygons is either empty or one point or a line segment, and the intersection of two edges is either empty or one vertex. Similarly, one can talk about polyhedral immersions of surfaces.

To get a definition of tight smooth surfaces consider the embedded surface in $\mathbb{R}^{3} / \Gamma_{1}$ shown in Figure 2(a). Obviously, Figure 2(a) shows a smooth and polyhedral embedding of the two-dimensional torus in $\mathbb{R}^{3} / \Gamma_{1}$ as is seen in Figure 2(b). It is a surface consisting only of points of vanishing curvature. (This is not possible in Euclidean three-space). Therefore, the lower bound for the total absolute curvature of a surface in this $C E S$ is zero and not 2. The example shows that there are surfaces for which the minimal value is reached. Because Gauss-Bonnet's theorem only depends on intrinsic measures, it is also valid in our case, and therefore it holds for a smooth surface $M$ in $C E S$ that

$$
\tau(M)=\int_{M} \frac{|K| d o}{2 \pi} \geq\left|\int_{M} \frac{K d o}{2 \pi}\right|=|\chi(M)|
$$

where $\chi(M)$ denotes the Euler characteristic of $M$. 


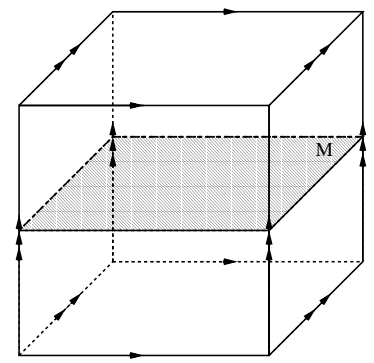

(a) Embedded

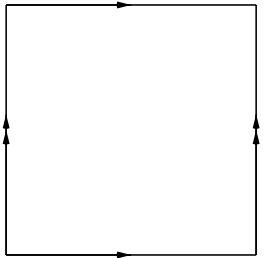

(b) Standard two-torus $T^{2}$ two-torus in $\mathbb{R}^{3} / \Gamma_{1}$

Figure 2. A first example

The first example shows that inequality (2.1) is sharp and we are now able to define tight smooth surfaces in $C E S$ in analogy to the definition in Euclidean three-space.

Definition 2.1. Let $f: M \rightarrow \mathbb{R}^{3} / \Gamma$ be a smooth immersion of a surface $M$ in a $C E S . f$ is called tight if the following equality holds:

$$
\tau(M)=|\chi(M)| .
$$

Conclusion 2.2. A surface $M$ is tight if and only if its Gaussian curvature $K$ does not change sign.

Proof. If $K$ does not change sign, $\int_{M}|K| d o=\left|\int_{M} K d o\right|$. Therefore, equality occurs in (2.1) and equation (2.2) holds for $M$. Conversely, it is true that

$$
|\chi(M)|=\left|\int_{M} \frac{K d o}{2 \pi}\right|=\tau(M)=\int_{M} \frac{|K| d o}{2 \pi},
$$

which implies that $K$ does not change sign.

It follows that an immersion $f: M \rightarrow \mathbb{R}^{3} / \Gamma$ of a surface $M$ with negative Euler characteristic $\chi(M)$ in any $C E S$ is tight if and only if there exists no point $p \in f(M)$ with positive curvature. If $\chi(M)$ is positive, $f$ is tight if and only if no point $p \in f(M)$ with negative curvature exists. In the case of $\chi(M)$ being zero, every point has to have vanishing curvature.

Remark 2.3. Tightness of surfaces in Euclidean three-space is also studied in terms of height functions: tightness is equivalent to the property that any non-degenerate height function has exactly one maximum on $f(M)$. In our case tightness for $\chi(M) \leq 0$ is equivalent to the property that no "height function" has a maximum on $f(M)$.

Furthermore, tightness of surfaces in Euclidean three-space is equivalent to the two-piece-property (TPP), meaning that any plane cuts $M$ into at most two pieces. In the case of $C E S$ as ambient space this is no longer true. The example given in Figure 2 has in some sense a one-piece-property. In fact, this one-piece-property implies tightness here whereas the converse direction is false. It seems to be an interesting problem to further study an analogous $k$-piece-property in the context of tightness in $C E S$. This seems to involve the fundamental group of the surface. More precisely, the question is how this fundamental group is mapped into the fundamental group of the $C E S$. 


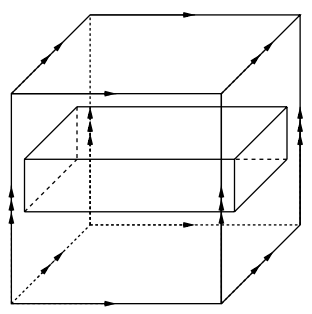

(a) Polyhedral $T^{2}$ in $T^{3}$

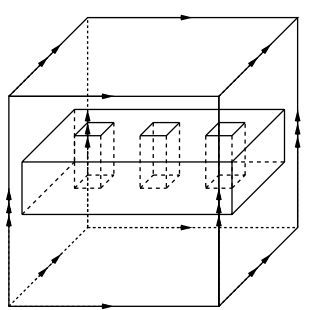

(b) Surface with $g=4$ in $T^{3}$

FIgURE 3 . Tight polyhedral immersions in $T^{3}$

If we consider polyhedral surfaces, it is necessary to redefine the total absolute curvature for this case as done in [5]. With this definition and the fact that GaussBonnet's theorem also exists for polyhedral surfaces (see [1] and 2]), it is possible to prove equivalent statements in terms of the polyhedral notation as done above for smooth surfaces. Therefore, we have the opportunity to deal with surfaces smooth or polyhedral - in the same way and we will do this below.

\section{EXISTENCE AND NON-EXISTENCE RESUlts}

To begin, we mention two tools which are necessary to prove the existence theorems. The first one is tight surgery. This is a well-known procedure used to construct tight examples of higher genus. This works for $C E S$ just as it does in three-space. We only have to beware of the identifications in the CES. The second one is tight smoothing as defined in [12. There it is shown that it is possible to smooth tight polyhedral surfaces if the vertices have valence three or are standardsaddle-vertices so that the resulting smooth surface has the same topological type and is also tight. This procedure can be transferred in $C E S$ without change.

Theorem 3.1. Let $M$ be an orientable surface. Then there exists a tight embedding $f: M \rightarrow \mathbb{R}^{3} / \Gamma$ in any given $C E S$. The map $f$ can be chosen to be smooth or polyhedral.

Proof. Every boundary of a three-dimensional convex body that does not meet the "boundary" of the fundamental domain of the different groups $\Gamma$ is a tightly embedded two-sphere. It is clear that no point of negative curvature exists, that the embedding can be chosen to be smooth or polyhedral and that it exists in every $C E S$.

Consider Figure 3(a). It shows a polyhedral two-dimensional torus in $\mathbb{R}^{3} / \Gamma_{1}$ (the three-dimensional torus $T^{3}$ ) which can easily be smoothed tightly because it has no vertex. To construct tight surfaces of higher genus, one has to attach handles as shown in Figure 3(b) which is an example of tight surgery. The additional handles carry no positive curvature with the result that the polyhedral surface is tight. Because the vertices which arose from the operation have the right valence, the polyhedral surface can be smoothed tightly; so we are able to construct tight surfaces - smooth or polyhedral - of any genus by attaching handles in the manner described.

To prove that this construction also works for the other $C E S$, we have to find opposite faces in the fundamental domain that are identified by a translation only. 


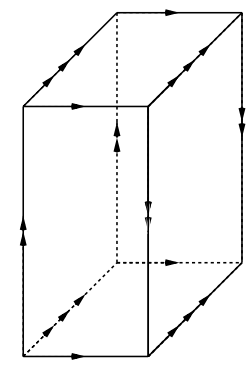

(a) $\mathbb{R}^{3} / \mathfrak{B}_{1}$

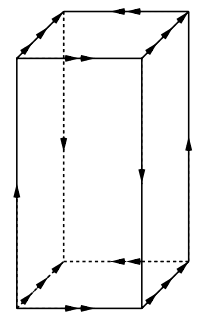

(c) $\mathbb{R}^{3} / \mathfrak{B}_{3}$

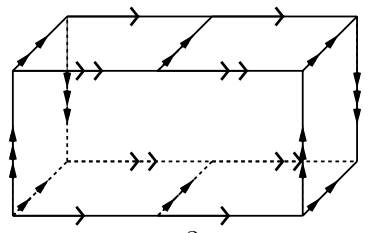

(b) $\mathbb{R}^{3} / \mathfrak{B}_{2}$

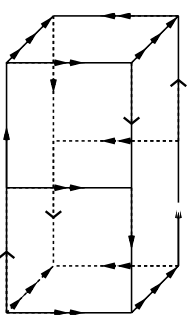

(d) $\mathbb{R}^{3} / \mathfrak{B}_{4}$

Figure 4. Models of the non-orientable $C E S$

Then we have the opportunity to start with the tightly embedded torus and follow the construction as above. If we study the fundamental domains corresponding to the orientable $C E S$ shown in Figure 1 and in [1], we find opposite faces that are identified by a translation only. To see this in the non-orientable case we look at Figure 4 based on the group actions of [10] showing models of the non-orientable $C E S$ where the arrows mark the way the edges are identified. They induce the face identifications in the same way as in Figure 1. In all four fundamental domains, there are opposite faces identified by a translation and the above construction works also in this case.

Of course, one can imagine many different tight orientable surfaces in $C E S$ which are not part of the construction used in the proof. Figure [5]shows some examples.

Theorem 3.2. Let $M$ be the Klein bottle. Then there exists a tight embedding - smooth or polyhedral - $f: M \rightarrow \mathbb{R}^{3} / \Gamma$ in every non-orientable $C E S$ and an immersion - smooth or polyhedral - $f: M \rightarrow \mathbb{R}^{3} / \Gamma_{i}, \quad i=2,4,5,6$, in all the orientable CES but the three-torus and $\mathbb{R}^{3} / \Gamma_{3}$. In the case of the CES being $\mathbb{R}^{3} / \Gamma_{2}$ or $\mathbb{R}^{3} / \Gamma_{6}, f$ can be chosen to be an embedding.

Proof. Consider Figure 6(a). It shows a smooth and polyhedral tight embedding of the Klein bottle in $\mathbb{R}^{3} / \mathfrak{B}_{1}$ because of the twist identification of the left and the right edge of $M$ and the identification with no twist of the top and bottom edge at the same time.

To see that the same is true for $\mathbb{R}^{3} / \mathfrak{B}_{2}$, we have to study the immersion $f: M \rightarrow$ $\mathbb{R}^{3} / \mathfrak{B}_{2}$ shown in Figure 6 (b). Because of the non-orientable identification of the front and the back face and the orientable identifications on the top and the bottom face, at the same time this is an immersion of the Klein bottle in $\mathbb{R}^{3} / \mathfrak{B}_{2}$. We can 


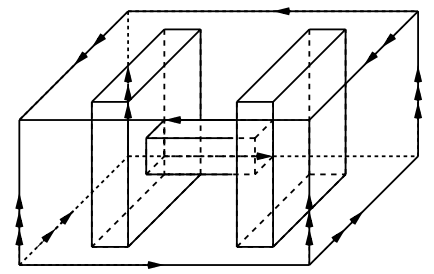

(a) Tightly immersed surface of genus two in $\mathbb{R}^{3} / \Gamma_{2}$

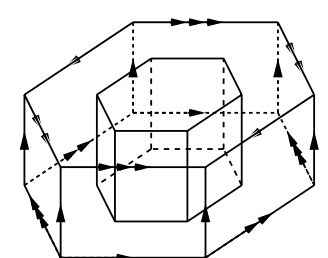

(b) Tightly immersed 2-torus in $\mathbb{R}^{3} / \Gamma_{3}$

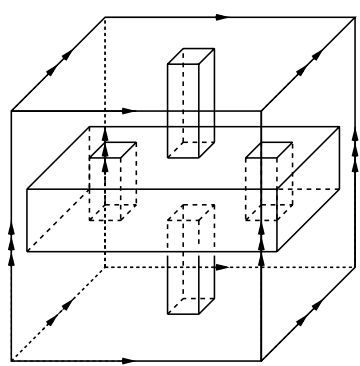

(c) Tightly immersed surface of genus 4 in $\mathbb{R}^{3} / \Gamma_{1}$

Figure 5. Some examples
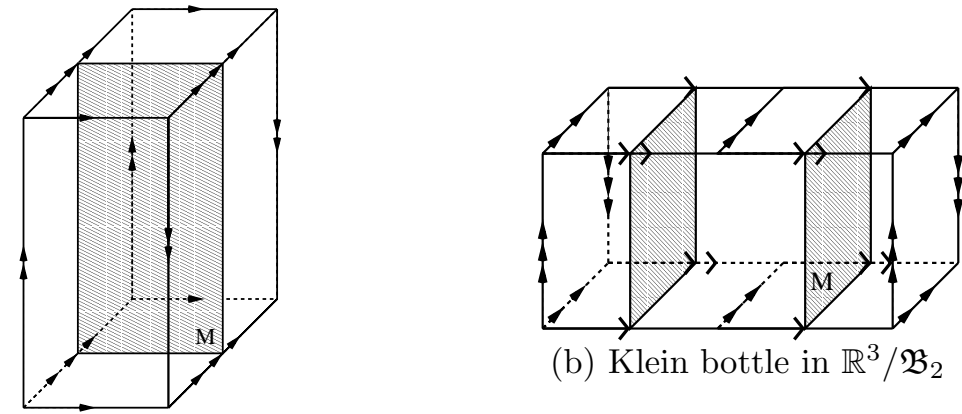

(b) Klein bottle in $\mathbb{R}^{3} / \mathfrak{B}_{2}$

(a) Klein bottle in $\mathbb{R}^{3} / \mathfrak{B}_{1}$

Figure 6. Embedded Klein bottle

look at it as a kind of hexagonal version of the Klein bottle shown in Figure 9 (see discussion below).

The cases of $\mathbb{R}^{3} / \mathfrak{B}_{3}$ and $\mathbb{R}^{3} / \mathfrak{B}_{4}$ work as shown in Figure 6 (a). The fundamental domains of these space forms have an orientable identification of the top and the bottom face and a non-orientable identification of the left and the right face (see Figure 44) so that the example of an immersion of the Klein bottle in $\mathbb{R}^{3} / \mathfrak{B}_{1}$ is one in $\mathbb{R}^{3} / \mathfrak{B}_{3}$ and $\mathbb{R}^{3} / \mathfrak{B}_{4}$. This proves the theorem for all the non-orientable $C E S$ because these maps are obviously embeddings.

In the case of $\mathbb{R}^{3} / \Gamma_{2}$, look at the surface $M$ embedded in this space form shown in Figure 7. The rotation by $\pi$ identifies the top and the bottom edges of the 


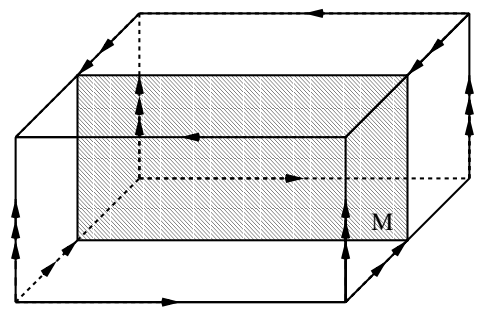

FiguRE 7 . Klein bottle in $\mathbb{R}^{3} / \Gamma_{2}$

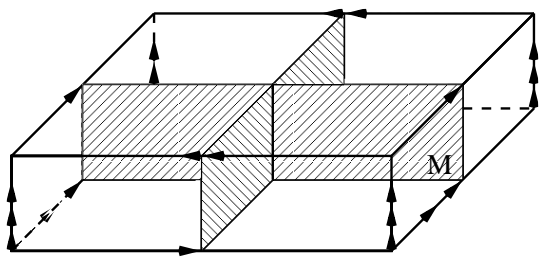

(a) Klein bottle in $\mathbb{R}^{3} / \Gamma_{4}$

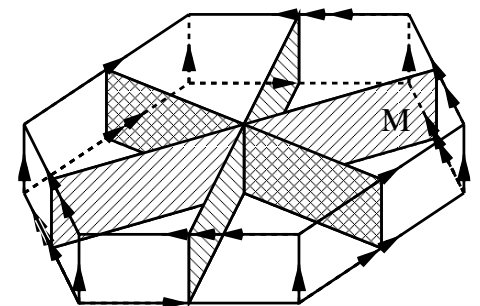

(b) Klein bottle in $\mathbb{R}^{3} / \Gamma_{5}$

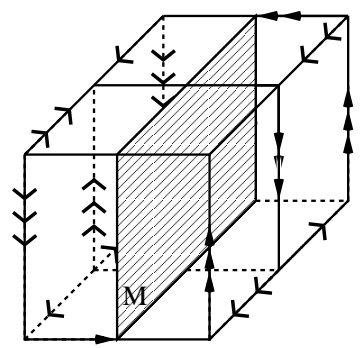

(c) Klein bottle in $\mathbb{R}^{3} / \Gamma_{6}$

Figure 8 . The Klein bottle in $\mathbb{R}^{3} / \Gamma_{4}, \mathbb{R}^{3} / \Gamma_{5}$ and $\mathbb{R}^{3} / \Gamma_{6}$

embedded surface with a twist, while the other two are identified with no twist. The resulting surface is therefore an embedded Klein bottle.

This idea is now extended to the remaining cases of the $C E S$ being $\mathbb{R}^{3} / \Gamma_{4}$, $\mathbb{R}^{3} / \Gamma_{5}$ and $\mathbb{R}^{3} / \Gamma_{6}$. The resulting surfaces are shown in Figure 8 . Note that in the case of $M$ immersed in $\mathbb{R}^{3} / \Gamma_{4}$ (Figure $8(\mathrm{a})$ ) the rotation of $\pi / 2$ attaches the top of one to the bottom of the other "plane", and passing through both the net effect is a rotation of $\pi$, i.e., a connection with a twist. The vertical edges are attached without twists, so $M$ is a Klein bottle. The same has to be done in the case of $\mathbb{R}^{3} / \Gamma_{5}$ to show that the immersed $M$ shown in Figure $8(\mathrm{~b})$ is a Klein bottle.

Note that the $M$ in $\mathbb{R}^{3} / \Gamma_{6}$ of Figure 8 (c) is an embedded surface. To see that it is a Klein bottle we have to study the edge identifications. The top "edge" is attached to the bottom as two separate edges, each with a twist. The front half of the top is attached to the front half of the bottom with a twist, while the back halves are also connected with a twist. The vertical edges are identified with a translation. Therefore, the result is a kind of hexagonal version of the Klein bottle shown in Figure 9 and the theorem is proved. 


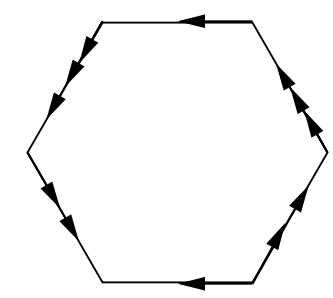

Figure 9. A hexagonal version of the Klein bottle

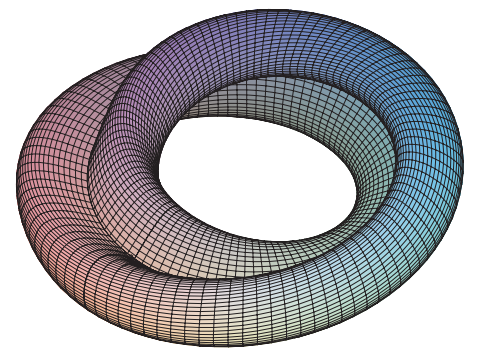

Figure 10. Klein bottle

Remark 3.3. There is another way of proving the theorem in the case of the orientable $C E S$ getting tight immersions of the Klein bottle. We start with the immersion of the Klein bottle in three-space shown in Figure 10. We can produce an equivalent immersion in $\mathbb{R}^{3} / \Gamma_{2}$ and $\mathbb{R}^{3} / \Gamma_{6}$ because they each have a pair of faces identified by a rotation of $\pi$ (see Figure 1). A simple cylinder over a figure eight is the immersion we are looking for. Figure 11(a) shows the solution while the top and bottom faces are identified by a rotation of $\pi$. To see that this is actually a tightly immersed Klein bottle, one can look at the polyhedral realization in Figure 11(b). Obviously, the total absolute curvature vanishes because the surface has no vertices. However, to compute its Euler characteristic we do not eliminate the edges and vertices on the top and bottom, but count them with their identifications. Now there are eight faces, eight vertical edges, eight horizontal edges and eight vertices. This correctly gives $\chi(M)=0$. With this, the surface satisfies equality (2.2) and is therefore tight. The non-orientability follows from the identification with the twist. This proves that the example shown is a tightly immersed Klein bottle as claimed.

Now let $\mathbb{R}^{3} / \Gamma_{4}$ be the $C E S M$ is immersed into. Figure 1 d) shows the fundamental domain of $\Gamma_{4}$ and the identifications on it.

The idea is similar to the case of $\mathbb{R}^{3} / \Gamma_{2}$. There, the solution was a cylinder over a figure eight. We extend this idea and allow another self-intersection on the screwline and take into consideration the identification by a rotation of $\pi / 2$ of the bottom and the top faces of the fundamental domain.

Figure 12(a) shows two figure eights covering each other after a rotation around the self-intersection of $\pi / 2$. Cylinders over these two figure eights are an example of a tightly immersed Klein bottle in $\mathbb{R}^{3} / \Gamma_{4}$ since the bottom and top faces are identified by a rotation of $\pi / 2$. 

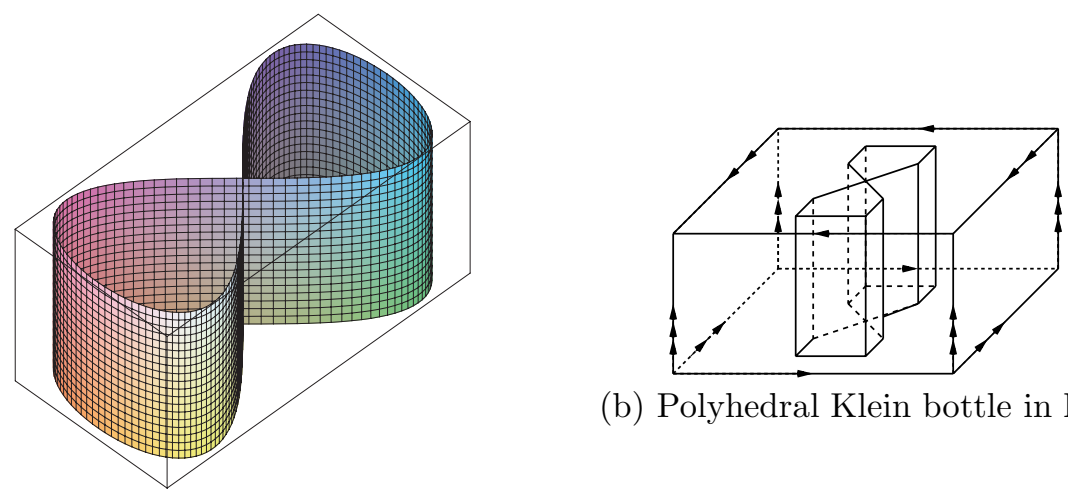

(b) Polyhedral Klein bottle in $\mathbb{R}^{3} / \Gamma_{2}$

(a) Smooth Klein bottle in $\mathbb{R}^{3} / \Gamma_{2}$

Figure 11. Klein bottle in $\mathbb{R}^{3} / \Gamma_{2}$

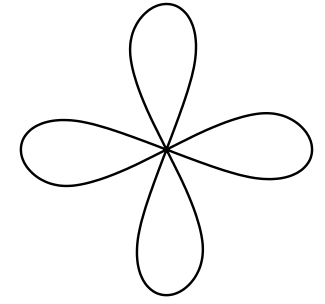

(a) "Base curve" for $\mathbb{R}^{3} / \Gamma_{4}$

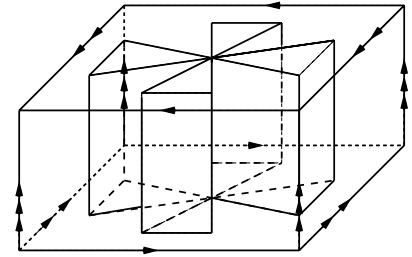

(b) Polyhedral realization

Figure 12 . The Klein bottle in $\mathbb{R}^{3} / \Gamma_{4}$

The total absolute curvature is obviously zero, so we are done if we can prove that the example is a closed surface, that it has vanishing Euler characteristic and that it is non-orientable.

It is easy to see that the example is a closed surface. There exists no boundary because of the rotational symmetry of the cylinder of $\pi / 2$ which coincides with the face identifications. For a better understanding of the example and to see that it has vanishing Euler characteristic look at Figure 12(b) which shows a polyhedral realization. Again, we have eight faces, eight vertical edges, eight horizontal edges and eight vertices, giving $\chi(M)=0$.

After twice the identification by the rotation of $\pi / 2$, we have the twist we need to get a non-orientable surface and therefore a Klein bottle.

The polyhedral surface has no vertices, so we can smooth it tightly and the result looks like cylinders over the two figure eights in Figure 12(a).

The same argument works with $\mathbb{R}^{3} / \Gamma_{5}$. Figure $\mathbb{1}(\mathrm{e})$ shows the fundamental domain and the identifications on it marked by the arrows. Cylinders over three figure eights which cover each other after a rotation of $\pi / 3$ are the solution. This is the surface we are looking for. Figure 13 shows the "base-curve" of the smooth immersion. The top and the bottom face of $\mathbb{R}^{3} / \Gamma_{5}$ are identified by a rotation of $\pi / 3$; therefore, the same argument as in the case of $\mathbb{R}^{3} / \Gamma_{4}$ is valid and we found an example of the Klein bottle tightly immersed in $\mathbb{R}^{3} / \Gamma_{5}$. 


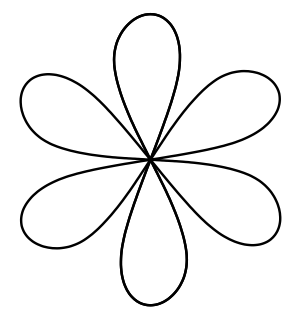

Figure 13. The "base curves" in $\mathbb{R}^{3} / \Gamma_{5}$

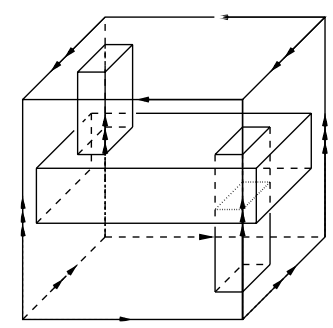

FiguRE 14. Tight torus with a non-orientable handle added in $\mathbb{R}^{3} / \Gamma_{2}$

Additional examples of tightly immersed non-orientable surfaces in non-orientable $C E S$ are given as part of the proof of Theorem 3.4 .

Theorem 3.4. Let $M$ be a non-orientable surface with even Euler characteristic $\chi(M)<0$. Then there exists a tight embedding - smooth or polyhedral $-f: M \rightarrow$ $\mathbb{R}^{3} / \Gamma$ in any $C E S$.

Proof. Let the $C E S$ be non-orientable. Consider a non-orientable identification of the left and right face in Figure 3(a). In every non-orientable $C E S$ there exist opposite faces which are identified non-orientable (see Figure 4). Then Figure 3(a) shows a tightly embedded polyhedral Klein bottle which can easily be smoothed tightly. Handles can be added as shown in Figure 3(b) and we have tight embeddings - smooth or polyhedral - of every $M$ with even Euler characteristic $\chi(M)<0$ in every non-orientable $C E S$.

In the case of orientable $C E S$ the following construction can be used. Start with a tightly embedded torus and glue a non-orientable handle into it so that the resulting surface is tight. Adding handles, as usual, proves the theorem. Consider the polyhedral immersion shown in Figure 14. The top and bottom faces are identified by a rotation of $\pi$ and the self-intersection is drawn in dotted lines. This is a tightly immersed Klein bottle with one handle which can be smoothed tightly. By adding handles, as in the orientable case, we can produce tightly immersed surfaces in $\mathbb{R}^{3} / \Gamma_{2}$ with any even Euler characteristic $\chi(M)<0$. This procedure works in every orientable $C E S$ except the three-torus. In the case of $\mathbb{R}^{3} / \Gamma_{1}$ the above construction has to be modified because it would produce singularities. To omit these we only have to glue the tight torus with a sheared handle. It can easily be shown that this sheared handle also carries no positive curvature so that the resulting surface is tight. Additional handles can be added as always. This construction leads to tight immersions because of the self-intersection. 


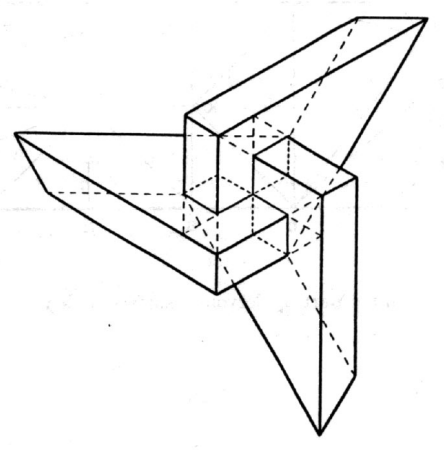

FiguRE 15. Inner part of a tightly immersed projective plane with two handles

To obtain tight embeddings in $\mathbb{R}^{3} / \Gamma_{i}, i=1,3,4,5$, we verify the above construction in the following way. We start with a tightly embedded flat torus as shown in Figure 2(a). This is possible in all the four CES. Attaching a vertical handle as in Figure 14 makes the result non-orientable which can easily be seen. As in the above construction we have to add a sheared handle in $\mathbb{R}^{3} / \Gamma_{1}$ to avoid singularities. Attaching handles, as usual, leads to embeddings of any higher genus as wanted. We remark that this construction also works in $\mathbb{R}^{3} / \Gamma_{2}$ but we use another construction in this case in the following.

To obtain embeddings of non-orientable surfaces $M$ with even Euler characteristic $\chi(M)<0$ in $\mathbb{R}^{3} / \Gamma_{2}$ and $\mathbb{R}^{3} / \Gamma_{6}$, we start with the embeddings of the Klein bottle in these $C E S$ shown in the proof of Theorem 3.2. Attaching a sheared handle to the embedded Klein bottle in $\mathbb{R}^{3} / \Gamma_{2}$ as in the case of $T^{3}$ produces an embedding of the non-orientable surface with $\chi(M)=-2$ in this $C E S$. Now handles can be attached as usual to produce examples of higher genus in this space form. Attaching a handle to the embedding of the Klein bottle in $\mathbb{R}^{3} / \Gamma_{6}$ is an example of the surface with $\chi(M)=-2$. Attaching more handles as usual proves the theorem.

Theorem 3.5. Let $M$ be a surface with odd Euler characteristic $\chi(M) \leq-5$. Then there exists a tight immersion - smooth or polyhedral $-f: M \rightarrow \mathbb{R}^{3} / \Gamma$ into any $C E S$.

Proof. The proof takes advantage of Kühnel and Pinkall's article 12 in which the authors give an example of a tightly immersed polyhedral projective plane with two handles which can be smoothed tightly. This surface $M_{\text {old }}$ can be divided into an inner part $I$ and a tetrahedron, the outer part. Figure [15] shows the Boy surface $I$. The handles that are glued into the tetrahedron in $M_{\text {old }}$ have to be modified so that every handle could be closed by a plane perpendicular to the bottom of the tetrahedron. Figure 16(a) shows the modification, while the arrows show the way the handles are "stretched". The result is shown in Figure 16(b).

This Boy surface is now glued into a tightly immersed torus. A triangular cylinder between opposite faces identified by a translation is such a torus. It is shown in Figure 16 by a dotted triangle. This immersion can be found in every $C E S$ because 


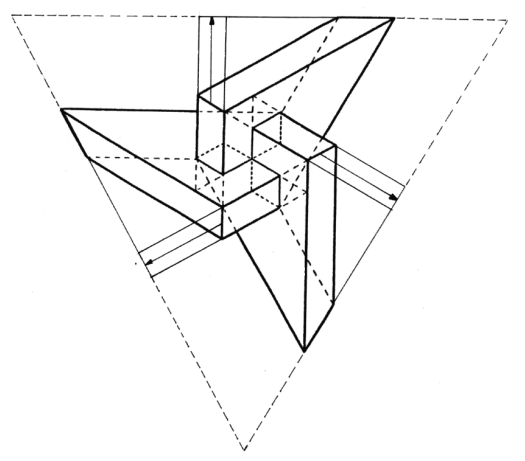

(a) Modification

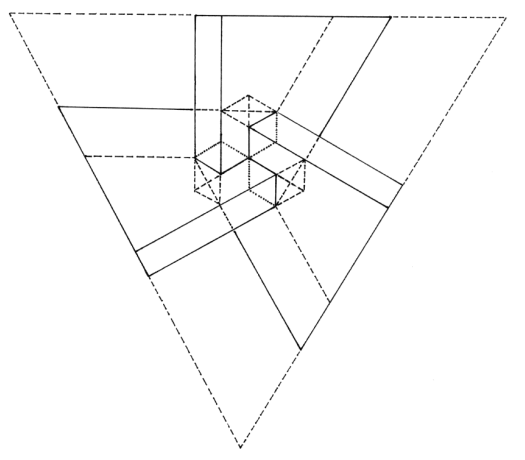

(b) Modified $I$ in a tight torus

FiguRE 16. A tight polyhedral projective plane with three handles

in every $C E S$ opposite faces exist which are identified by a translation (see Figure 1 and Figure 4).

We see that its Euler characteristic is equal to -5 by the following. The inside of the newly created surface is a Boy surface with three holes and has therefore $\chi=-2$. The triangular torus with three disks removed give $\chi=-3$. If we glue this together we get $\chi(M)=-5$. The fact that the surface is closed can easily be seen with the result that the present surface is a projective plane with three handles. Now, we have to show that the constructed surface is tight. Therefore, we look at the total absolute curvature of $M_{\text {old }}$,

$$
\tau\left(M_{\text {old }}\right)=4-\chi\left(M_{\text {old }}\right)=4-(-3)=7 .
$$

The tetrahedron carries a total absolute curvature of 2 and the torus in the new surface zero. Thus, the following is true:

$$
\tau\left(M_{\text {new }}\right)=4-\chi\left(M_{\text {old }}\right)-2=4-(-3)-2=\left|\chi\left(M_{\text {new }}\right)\right| .
$$

This shows the tightness of the constructed surface.

The old surface is tightly smoothable. Similarly, the new one is also, because the vertices involved in the modification do not change their valence nor do they produce saddle points. Thus the requirements for the tight smoothing remain valid and the new surface is tightly smoothable. 


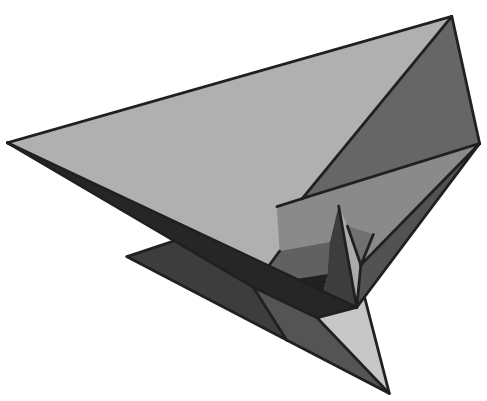

FiguRE 17. The inside of the polyhedral real projective plane with one handle

More handles can be attached in the known way and we obtain tight immersions - smooth or polyhedral - of all non-orientable surfaces with odd Euler characteristic $\chi(M) \leq-5$ in all $C E S$.

Theorem 3.6. Let $M$ be the projective plane with two handles. Then there exists a tight polyhedral immersion $f: M \rightarrow \mathbb{R}^{3} / \Gamma$ into any $C E S$.

Proof. Consider the polyhedral surface constructed in [6] and [7]. It is a tight projective plane with one handle that is not smoothable. Figure 17 il shows the "inside" of this surface, the projective plane with two holes. If we glue this into a torus (immersed in the $C E S$ as shown in Figure 3(a)) with two holes, the resulting surface $M$ has Euler characteristic $\chi(M)=-3$ (projective plane with two holes $=-1$; a torus with two holes $=-2$ ). Therefore, it is a polyhedral projective plane with two handles. Now we have to show that $M$ is tightly immersed. Positive curvature can only appear at vertices that are involved in the glueing, because the surface we start with together with a part of its convex envelope is tight and therefore the vertices that carry positive curvature lie on the boundary of the convex hull. There are seven of these which we have to study exactly. We see that the positive curvature of each of these vertices change into negative curvature because of our glueing. So the resulting surface only consists of negative curvature and it is therefore tight but not smoothable by the known process because of the high valence of a vertex "inside" [6].

Remark 3.7. Although there exists a smooth immersion of the surface $M$ with $\chi(M)=-3$ in $\mathbb{E}^{3}$ and the polyhedral solution above, it seems to be difficult to find a smooth immersion of $M$ into a $C E S$. All the studied realizations carried positive curvature and it seems to be possible that the polyhedral and the smooth theories differ here in contradiction to the classical theory where they differ for the surface with $\chi(M)=-1$.

Theorem 3.8. There exists no tight immersion of the projective plane $M$ in any $C E S$.

Proof. Assume that there is a tight immersion of the projective plane $M$ in any $C E S$.

\footnotetext{
${ }^{1}$ Reproduced from URL: http://www.math.union.edu/locate/rp2-handle/ with the kind permission of Davide P. Cervone.
} 
Let $\pi$ denote the Riemannian covering map $\pi: \mathbb{R}^{3} \rightarrow \mathbb{R}^{3} / \Gamma$. Note that $\pi$ is a local isometry and that $\pi^{-1}(M)$ must be isomorphic to a disjoint collection of twospheres or projective planes because the surface $M$ has to be covered by surfaces and these two kinds are the only possible ones. Because $\pi$ is a local isometry, each of these spheres or projective planes must have locally the same curvature properties as $M$, meaning that they have to consist of points with non-negative curvature only. This is obviously not possible for projective planes. The covering by spheres of a projective plane is two-sheeted in each component, so the total absolute curvature of each sphere must be equal to $4 \pi$. Furthermore, the spheres are convex and embedded (see [9]). To have a projective plane in the quotient, there has to be a non-trivial element of order two in the group of deck transformations, because the fundamental group of a projective plane is isomorphic to $\mathbb{Z}_{2}$. But there is no non-trivial element of finite order in each $\Gamma$ (see [16]), the deck transformation groups of the coverings, with the result that $M$ cannot be tightly immersed in any $C E S$.

Theorem 3.9. There exists no tight immersion of the Klein bottle $M$ neither in the three-torus $\mathbb{R}^{3} / \Gamma_{1}$ nor in $\mathbb{R}^{3} / \Gamma_{3}$.

Proof. If $M$ would admit a tight immersion in $\mathbb{R}^{3} / \Gamma_{1}$, it must have vanishing Gaussian curvature $K$ because of the definition of tightness. Let $\pi$ denote the Riemannian covering map $\pi: \mathbb{R}^{3} \rightarrow \mathbb{R}^{3} / \Gamma_{1}$ and $M^{\prime}=\pi^{-1}(M)$. Note that $\pi$ is a local isometry and that $M$ is connected and complete. This implies the completeness of $M^{\prime}$. With this and the vanishing Gaussian curvature on $M^{\prime}$ it follows that $M^{\prime}$ is a collection of cylinders or a collection of planes [15]. By definition, a cylinder is a regular surface $S$ such that through each point $p \in S$ there passes a unique line $R(p) \subset S$ (the generator through $p$ ) which satisfies the condition that if $q \neq p$, then the lines $R(p)$ and $R(q)$ are parallel or equal. Therefore, one can look at $M^{\prime}$ as being a collection of cylinders because a plane is just a special cylinder by this definition.

For $M$ being a Klein bottle in $\mathbb{R}^{3} / \Gamma_{1}$, we need a Möbius strip around a generating element of the fundamental group of $M$. We will prove that this is not possible.

Let $c$ be a loop in the fundamental group of $M$ which is not null-homotopic and $\tilde{c} \subset \pi^{-1}(c)$ the lift of this loop traversed once into $\mathbb{R}^{3}$.

Note that the tangent in the "starting point" and the tangent in the "end point" of $\tilde{c}$ are parallel because the elements of the deck transformation group are translations only. Furthermore, notice that $M^{\prime}$ is orientable because it is cylindrical as stated above. With this orientation and the orientation of $\tilde{c}$ we are able to obtain an orientated suitable small $\varepsilon$-neighborhood $C_{\tilde{c}}$ of $\tilde{c}$. Note that $C_{\tilde{c}}$ is an oriented strip which maps under $\pi$ to such an oriented cylinder strip in $\mathbb{R}^{3} / \Gamma_{1}$ because the group of deck transformations only contains translations and identify therefore the parallel tangents of the starting and the end points orientably. This implies that there exists no tight immersion of the Klein bottle in $\mathbb{R}^{3} / \Gamma_{3}$, because the lift of such an immersion in $\mathbb{R}^{3} / \Gamma_{1}$ would have to be either tori or Klein bottles. The first is impossible because the covering is 3 -sheeted, the second because of the proof above.

\section{EXAMPLES}

(1) There exists a tight immersion — smooth or polyhedral — of the torus into any $C E S$ that is not an embedding (i.e., that has double points). 
This is also true if the torus is immersed in $\mathbb{E}^{3}$ (see [3], Example 1.3.5). To produce such an immersion in a $C E S$ we use the idea of a tube over a figure-eight that was introduced in Remark [3.3] and Figure 11] If we have the immersion shown in Figure 11 in $T^{3}$, top and bottom faces of the fundamental domain are identified by a translation. The Euler characteristic does not change. The only thing we have lost is the non-orientability. The example is therefore a tight torus with a self-intersection line. This immersion exists in every $C E S$ because the required identification of faces exists in every $C E S$ as stated above.

(2) The following operation is described in 8. Start with a tight orientable polyhedral surface that has a monkey saddle. Then it is possible to replace this monkey saddle by a piece of Brehm's projective plane [4 and the resulting surface is still tight but non-orientable. The author constructs a torus containing a monkey saddle and having two parallel faces at the top and bottom. Remove these and glue the resulting surface into a torus shown in Figure 3 with two holes and replace the monkey saddle by the Brehm's projective plane to get a tight polyhedral surface with Euler-characteristic $\chi(M)=-5$. This surface is not smoothable with the known procedure because of the high valence of some vertices. This does not say that it is not smoothable at all.

\section{Questions}

Is there a tight immersion of the projective plane with one handle in any $C E S$ ?

We think that the answer is negative, because it cannot be realized in the interior of the $C E S$ and the identifications may induce a handle with the result that a tight surface of this type cannot exist.

Is there a tight and smooth immersion of the projective plane with two handles in any $C E S$ ?

As in the previous case, we think that the answer is negative. The reasons are very much the same as above with the addition that no projective plane with two holes consisting only of points with negative curvature exists in Euclidean threespace. See also Remark 3.7 .

\section{REFERENCES}

[1] Thomas F. Banchoff, Tightly embedded 2-dimensional polyhedral manifolds, Amer. J. Math. 87 (1965), 462-472. MR 31:2729

[2] Critical points and curvature for embedded polyhedral surfaces, Amer. Math. Monthly 77 (1970), 475-485. MR 41:4444

[3] Thomas F. Banchoff and Wolfgang Kühnel, Tight submanifolds, smooth and polyhedral, Tight and Taut Submanifolds (Thomas E. Cecil and Shiing-shen Chern, eds.), Mathematical Sciences Research Institute Publications, vol. 32, Cambridge University Press, 1997, pp. 51-118. MR 99d:53063

[4] Ulrich Brehm, How to build minimal polyhedral models of the Boy surface, Math. Intell. 12 (1990), 51-56. MR 91h:52007

[5] Ulrich Brehm and Wolfgang Kühnel, Smooth approximation of polyhedral surfaces regarding curvatures, Geometriae Dedicata 12 (1982), 435-461. MR 84c:53056

[6] Davide P. Cervone, Tightness for smooth and polyhedral immersions of the projective plane with one handle, Tight and Taut Submanifolds (Thomas E. Cecil and Shiing-shen Chern, eds.), Mathematical Sciences Research Institute Publications, vol. 32, Cambridge University Press, 1997, pp. 119-134. MR 98j:53071.

[7] _ A tight polyhedral immersion in three-space of the projective plane with one handle, Pacific J. Math. 196 (2000), 113-122. MR 2001m:57038 
[8] _ A tight polyhedral immersion of the twisted surface of Euler characteristic -3, Topology 40 (2001), 571-584. MR 2002f:57035]

[9] Shiing-shen Chern and Richard K. Lashof, On the total curvature of immersed manifolds, II, Mich. Math. J. 5 (1958), 5-12. MR 20:4301

[10] W. Hantzsche and H. Wendt, Dreidimensionale euklidische Raumformen, Mathematische Annalen 110 (1935), 593-611.

[11] Wolfgang Kühnel, Differential geometry: curves - surfaces - manifolds, Student Mathematical Library, vol. 16, American Mathematical Society, 2002.

[12] Wolfgang Kühnel and Ulrich Pinkall, Tight smoothing of some polyhedral surfaces, Global Differential Geometry and Global Analysis 1984 (Berlin; Heidelberg; New York) (A. Dold and B. Eckmann, eds.), Lecture Notes in Mathematics, vol. 1156, Springer, 1984, pp. 227-239. MR 87h:53085

[13] Nicolaas H. Kuiper, On surfaces in Euclidean three-space, Bull. Soc. Math. Belg. 12 (1960), 5-22. MR 23:A609

[14] Convex immersions of closed surfaces in $E^{3}$, Comment. Math. Helv. 35 (1961), 85-92. MR 23:A2175

[15] William S. Massey, Surfaces of Gaussian curvature zero in Euclidean 3-space, Tôhoku Math. J. 14 (1962), 73-79. MR 25:2527

[16] Joseph A. Wolf, Spaces of constant curvature, 4th ed., Publish or Perish, Berkeley, 1977.

Department of Mathematics, University of Stuttgart, Pfaffenwaldring 57, 70550 Stuttgart, Germany

E-mail address: Otto@mathematik.uni-stuttgart.de 